\title{
Influencia de fuentes y concentraciones de saborizantes sobre el consumo de forraje en caprinos ${ }^{1}$
}

\author{
Influence of different sources and concentrations of flavors on the intake in \\ caprine $^{1}$
}

\author{
Claudio Fabián Vargas-Rodríguez², José Andrés Carvajal-Villalta ${ }^{3}$
}

\begin{abstract}
Resumen
El comportamiento de los caprinos a los estímulos gustativos afecta en el consumo de materia seca (MS), y la inclusión de estimuladores sensoriales puede incrementar la productividad. El objetivo de este experimento fue evaluar diferentes fuentes y concentraciones de saborizantes sobre el consumo de forraje en cabras. La investigación se desarrolló en la Estación Experimental de Ganado Lechero "Alfredo Volio Mata" de la Universidad de Costa Rica, en Ochomogo, Costa Rica, durante octubre del 2015. Una prueba de "cafetería" fue usada en un modelo de crossover en bloques. Pennisetum purpureum cv King grass mezclado con cuatro sabores (ácido, amargo, dulce y salado) en cinco diferentes concentraciones $(0 ; 0,25 ; 0,5 ; 0,75$ y $1,0 \%)$, fue suministrado a un grupo de cabras. Los caprinos mostraron mayor preferencia por el forraje mezclado con saborizante amargo, y menor preferencia por los sabores dulce y salado. El consumo de forraje en base seca que mostraron los animales cuando se aplicó el sabor ácido fue de $0,977 \mathrm{~kg} / \mathrm{d}$, mientras que, cuando se usaron los saborizantes dulce y salado fue de 0,855 y $0,822 \mathrm{~kg} / \mathrm{d}$, respectivamente. Estas diferencias fueron significativas. Los caprinos consumieron el $68,28 \%$ de la cantidad de forraje ofrecida cuando se usó el saborizante amargo, pero solo consumieron el 56,43\% de lo ofrecido cuando el forraje fue acidificado. Al comparar la inclusión de cualquier sabor con respecto a un tratamiento control sin saborizante, no se observaron diferencias significativas ni en el consumo de materia seca, ni el grado de aceptación. No se observó ningún efecto en el consumo de materia seca ni en el porcentaje de aceptación cuando se usaron diferentes concentraciones de los saborizantes. La interacción entre sabor y concentración de sabor no generó diferencias significativas en ninguno de los parámetros evaluados.
\end{abstract}

Palabras claves: cabras, consumo de piensos, estímulo, nutrición animal, pequeños rumiantes.

\begin{abstract}
The behavior of caprine to taste stimuli affects the consumption of dry matter (MS), and the inclusion of sensorial stimulators can increase productivity. The objective of this experiment was to evaluate different sources and concentrations of flavoring on forage intake in goats. The research was developed at the Dairy Cattle Experimental Station "Alfredo Volio Mata" of the University of Costa Rica, in Ochomogo, Cartago, Costa Rica during October

1 Recibido: 30 de marzo, 2017. Aceptado: 20 de febrero, 2018. Este trabajo formó parte del proyecto 737-A8-078 inscrito en la Vicerrectoría de Investigación. Universidad de Costa Rica, San José, Costa Rica.

2 Universidad de Costa Rica, Facultad de Ciencias Agroalimentarias, Estación Experimental Alfredo Volio Mata. fabian.vargas@ucr.ac.cr / cfabianv@gmail.com

3 Universidad de Costa Rica, Facultad de Ciencias Agroalimentarias, Escuela de Zootecnia. andzoot96@yahoo.com
\end{abstract}


2015. A coffee-shop test was used in a block crossover model. Pennisetum purpureum cv King grass mixed with four flavors (acid, bitter, sweet and salty) in five different concentrations $(0,0.25,0.5,0.75$ and $1.0 \%)$ was supplied to a group of goats. Goats showed the greater preference for forage mixed with a bitter flavor, and less preference for sweet and salty flavors. The forage intake on a dry basis showed by the animals when the acid flavor was applied, was $0.977 \mathrm{~kg} / \mathrm{d}$, when the sweet and salty flavorings were used the intake was 0.855 and $0.822 \mathrm{~kg} / \mathrm{d}$, respectively. These differences were significant. The goats consumed $68.28 \%$ of the amount of offered forage when the bitter flavor was used, but only consumed $56.43 \%$ of what was offered when the forage was acidified. When comparing the inclusion of any flavor with respect to an unflavored control treatment, no significant differences were observed in the consumption of dry matter or the degree of acceptance. No effect on dry matter consumption or acceptance percentage was observed when different concentrations of flavorings were used. The interaction between flavor and flavor concentration did not generate significant differences in any of the evaluated parameters.

Keywords: goats, feed consumption, stimulus, animal nutrition, small ruminants.

\section{Introducción}

Para incrementar la eficiencia productiva de un hato es necesario estimular el consumo de materia seca de los animales. Dentro de los factores que entran en juego para favorecer una mayor ingesta, se destacan las características propias del alimento (Olivares et al., 2012), como el sabor, la textura y el olor, las cuales desencadenan una respuesta sensorial que estimulan el apetito, lo que se conoce como palatabilidad del alimento (Baumont, 1996; Araujo y Milgram, 2004). Este fenómeno ha sido ampliamente estudiado y algunos de los trabajos sugieren que la palatabilidad se debe considerar al momento de formular raciones para animales en cualquier etapa productiva, $\mathrm{y}$ así obtener un mayor beneficio de las dietas (Miller-Cushon et al., 2014).

En el caso de los caprinos, el comportamiento ante los estímulos gustativos y las características inherentes de la especie, como la movilidad de sus labios y su capacidad selectiva (Animut et al., 2005), inciden enormemente en el consumo. Estos aspectos hacen necesario manejar todos los factores que puedan atraer más los animales a los comederos para aumentar el consumo, sobre todo del recurso forrajero. Algunos estudios han evaluado las respuestas a estímulos sensoriales; por ejemplo, De-Rosa et al. (2002) aplicaron extractos aromáticos a pellets de ryegrass (Lolium perenne, cv. Belida) y trébol blanco (Trifolium repens, cv. Huia), y observaron que los tratamientos aplicados incrementaron el consumo de materia seca de los caprinos. En otro estudio donde alimento peletizado fue tratado con saborizantes artificiales (fresa, naranja, manzana, caramelo, maple, cebolla, ajo y trufa), se observó un efecto positivo sobre el consumo en comparación con el tratamiento control (Robertson et al., 2006).

A pesar de las referencias disponibles, aún hay carencia de información cuantitativa sobre el uso de saborizantes para estimular el consumo de forraje en ganado caprino, especialmente en los sistemas de explotación en confinamiento. Los caprinos en estabulación mayoritariamente son alimentados con pastos de corte, los cuales son picados para facilitar la homogeneidad de las dietas, y disminuir el desperdicio. No obstante, este proceder modifica la conducta selectiva natural de los animales, que por sí mismos estimulan el consumo (Rutter, 2006). El objetivo de este experimento fue evaluar diferentes fuentes y concentraciones de saborizantes sobre el consumo de forraje en cabras.

\section{Materiales y métodos}

El experimento se ejecutó en el mes de octubre del 2015 en la Estación Experimental de Ganado Lechero Alfredo Volio Mata de la Universidad de Costa Rica. Este centro experimental se encuentra ubicado a 1542 msnm, 
en el Alto de Ochomogo, distrito de San Rafael del Cantón de la Unión, que pertenece a la provincia de Cartago, Costa Rica, a 955'10" latitud Norte y 8357'20” longitud Oeste. La zona muestra una precipitación media anual de $1466 \mathrm{~mm}$, distribuidos en la época lluviosa que va de mayo a noviembre, además, posee una humedad relativa media del $84 \%$ y una temperatura que va de $\operatorname{los} 13,5^{\circ} \mathrm{C}$ a $\operatorname{los} 23{ }^{\circ} \mathrm{C}$, con una temperatura media anual de $17,9^{\circ} \mathrm{C}$ (Pineda-Cordero et al., 2016). El suelo es de origen volcánico, clasificado como Typic Hapludands, caracterizado por poseer buen drenaje y una fertilidad media (Taxonomy-Soil, 2003).

\section{Manejo del componente forrajero}

La especie forrajera que se utilizó en este experimento fue Pennisetum purpureum cv King grass, la cual fue establecida en un área de $1056 \mathrm{~m}^{2}$. La edad de cosecha que se seleccionó para este trabajo fue de 75 días, momento en el cual, según indican experiencias anteriores, el material presenta una mejor digestibilidad y una mayor concentración de nutrientes (Chacón-Hernández y Vargas-Rodríguez, 2010). Para minimizar la variación de edad del King grass a lo largo del experimento, inicialmente se realizaron cortas de uniformización escalonadas cada quince días para lograr seis segmentos iguales de alrededor de $176 \mathrm{~m}^{2}$ cada uno. Inmediatamente después de la corta, se aplicó un herbicida a base de Ion Paraquat $\left(\mathrm{C}_{12} \mathrm{H}_{14} \mathrm{Cl}_{12} \mathrm{~N}_{2}\right)$ para realizar un control preventivo de malezas, con una dosificación de 2,5 1/ha (Márquez et al., 2007). El king grass se fertilizó con 115,5 kg/ha/año de nitrógeno (ArayaMora y Boschini-Figueroa, 2005), distribuidas en cada corta y aplicadas treinta días después de uniformizado.

Previo a iniciar el experimento, se tomaron tres muestras del king grass en diferentes sectores del cultivo seleccionados aleatoriamente, que fueron analizadas en el Laboratorio de Bromatología de la Estación Experimental de Ganado Lechero Alfredo Volio Mata, para conocer la composición nutricional del material (Cuadro 1). Las muestras se secaron en un horno a $55^{\circ} \mathrm{C}$ y se molieron en un molino con un tamaño de criba de 1-mm. Posteriormente, se determinó el contenido de materia seca colocando sub-muestras en un horno a $105^{\circ} \mathrm{C}$. El contenido de proteína cruda se determinó mediante el método de Kjeldahl (AOAC, 1984; método 7.033-7.037). La concentración de grasa se determinó por extracción con éter (AOAC, 2000; método 920.9); mientras que, la fracción de FDN (fibra en detergente neutro) se obtuvo en presencia de sulfito de sodio y amilasa, y la FDA (fibra en detergente ácido) en presencia de ácido sulfúrico, para ambos casos se utilizó un analizador de fibra (Van-Soest et al., 1991). En un horno a $500{ }^{\circ} \mathrm{C}$ se realizó la combustión de las muestras y así se determinó el contenido de cenizas de cada muestra (AOAC, 2000; método 942.05).

Cuadro 1. Descripción del contenido bromatológico del pasto Pennisetum purpureum cv. king grass cosechado a 75 días. Ochomogo, Cartago, Costa Rica. 2015.

Table 1. Description of the bromatological content of the pasture Pennisetum purpureum cv. king grass harvested at 75 days. Ochomogo, Cartago, Costa Rica. 2015.

\begin{tabular}{cc|cc}
\hline Nutriente, $\%$ & Valor & Nutriente, $\%$ & Valor \\
\hline Materia seca & 17,39 & Fibra en detergente ácido & 44,40 \\
Matera orgánica & 83,95 & celulosa & 38,60 \\
Proteína cruda & 6,56 & Hemicelulosa & 29,27 \\
Fibra en detergente neutro & 73,68 & Lignina & 5,80 \\
\hline
\end{tabular}




\section{Manejo de los animales}

Para este experimento se seleccionaron diez animales caprinos de las razas Saanen, Toggenburg y Lamancha, con un peso promedio de $50 \pm 2,1 \mathrm{~kg}$ y con una condición corporal de 3 en una escala de 1 a 5 (Gallego-Calvo et al., 2014), donde 1 son animales muy flacos y 5 animales muy gordos. Además, los animales fueron seleccionados con estado fisiológico y edades similares, sin partos previos y que no estuvieran gestantes.

Con el objetivo de asegurar el estado óptimo de los animales, se realizó un diagnóstico del estado sanitario empleando el método de campo FAMACHAC (Vargas-Rodríguez, 2007), que relaciona la coloración de la conjuntiva del ojo de los caprinos con la incidencia parasitaria. Con base en los resultados obtenidos, se ejecutó un programa correctivo de desparasitación con fenbendazol $(5,0 \mathrm{ml} / 100 \mathrm{~kg} \mathrm{PV})$. Al mismo tiempo, se midieron los niveles de hematocrito en sangre y se aplicaron $5 \mathrm{ml} /$ animal de un coadyuvante metabólico a base de fósforo orgánico y $1 \mathrm{ml} / 10 \mathrm{~kg}$ PV (siguiendo las recomendaciones del fabricante) de un complejo multivitamínico (vitamina B1, B3 y B6) para mejorar los niveles de hemoglobina del grupo experimental.

Los animales se dividieron en dos grupos y se colocaron en corrales con un área de $12 \mathrm{~m}^{2}$, con capacidad para albergar diez animales por corral, y la longitud de comedero por animal en cada corral fue de $40 \mathrm{~cm}$; sin embargo, solamente se colocaron cinco animales por aparto para darles mayor espacio de comedero por animal. Los comederos se adaptaron y se colocaron divisiones físicas provisionales de manera que los tratamientos no se mezclaran y pudieran afectar la prueba. Las divisiones de los comederos permitieron colocar los cinco tratamientos en cada comedero. Durante todo el experimento los animales tuvieron acceso ilimitado a agua. En la Figura 1 se observa el diagrama de la división de los comederos.

\section{Tratamientos y modelo experimental}

La metodología que se utilizó para alcanzar los objetivos planteados en este experimento fue una prueba de cafetería (Rangel et al., 2004), aplicada a un modelo de Crossover en bloques para medir el grado de aceptación del pasto Pennisetum purpureum cv King grass, picado a un tamaño de partícula de aproximadamente 2,5 cm y mezclado con cinco diferentes concentraciones de cinco diferentes sabores y ofrecidos a un grupo experimental de cabras.

La cantidad de forraje que se suministró a los animales fue determinada con base en información obtenida en investigaciones anteriores (Chacón-Hernández y Vargas-Rodríguez, 2010), donde se observó que los caprinos diariamente consumían la cantidad de materia seca equivalente al $0,52 \%$ de su peso vivo cuando se les ofreció king grass, cosechado a 75 días. Por lo tanto, se ofreció un total de $15 \mathrm{~kg}$ de pasto por grupo, se repartió dicha cantidad entre los cinco tratamientos y se fraccionó en tres tiempos de consumo por día. El alimento con los diferentes tratamientos se colocó en los comederos en posiciones distintas en cada tiempo de comida, seleccionadas aleatoriamente para evitar el efecto de acostumbramiento y que los animales tuvieron libre acceso al alimento para seleccionar según su agrado. El suministro de las dietas experimentales se realizó tres veces al día la primera exponiendo a los animales a la dieta durante una hora y dejando un lapso de 1,50 horas para recibir la siguiente oferta de alimento, en un periodo comprendido entre las 8:00 am y las 3:00 pm.

La mayoría de los estudios de palatabilidad son de corta duración, debido a que en estudios prolongados los efectos producidos son asociados con modificaciones digestivas (Baumont et al., 1996), por esta razón, los animales tuvieron acceso a las dietas experimentales por un espacio de una hora, y los periodos de prueba tuvieron una duración de cinco días, con dos repeticiones y un intervalo de dos días entre cada repetición. Pasadas las dos semanas con el primer sabor se dejó un periodo de descanso de siete días antes de iniciar el mismo procedimiento con el siguiente sabor. Durante esos siete días los animales recibieron la ración correspondiente sin la aplicación de ningún sabor. 


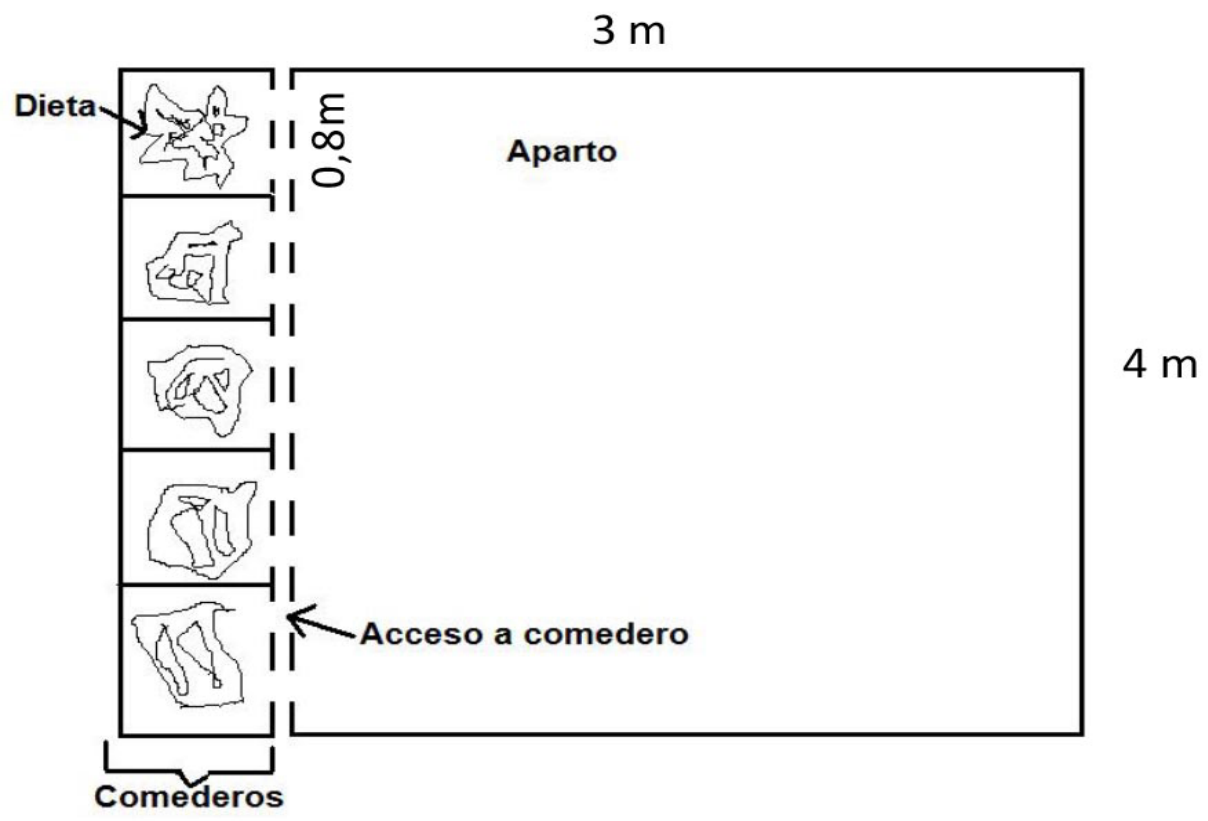

Figura 1. Ajuste de los comederos con divisiones físicas para separar los diferentes tratamientos y facilitar el acceso de las cabras a los tratamientos durante el experimento. Ochomogo, Cartago, Costa Rica. 2015.

Figure 1. Adjustments of feeders with physical divisions to separate different treatments and to facilitate access of the goats to treatments during the experiment. Ochomogo, Cartago, Costa Rica. 2015.

Para este experimento se evaluaron los sabores y las fuentes siguientes: amargo (saborizado con extracto comercial de café), dulce (obtenido a partir de una mezcla comercial de aspartame con acesulfame-k), ácido (saborizado con ácido cítrico), salado (cloruro de sodio) y un control, al cual solamente se le agregó agua. Estos aditivos fueron preparados en agua para obtener concentraciones de $0,00 \%, 0,25 \%, 0,50 \%, 0,75 \%$ y $1,00 \%$ en relación peso/peso, para lo cual se utilizó una balanza analítica AND modelo GR-300. Las soluciones se aplicaron en el king grass, rociadas con botellas de aspersión con capacidad de $960 \mathrm{ml}$. Antes de iniciar el estudio, se realizaron pruebas de calibración para asegurar un manejo adecuado de la descarga de las botellas y lograr las concentraciones deseadas de los tratamientos. De esta manera, se determinó que accionando el dispositivo setenta veces a su presión máxima, se lograba una descarga de $60 \mathrm{ml}$ de solución. La aplicación de los tratamientos no influenció la composición química del forraje ni la estructura física.

La aceptación y el consumo de materia seca se determinó pesando el material ofrecido y posteriormente, el material rechazado en cada tiempo de comida.

\section{Análisis estadístico}

Los datos obtenidos fueron analizados con el paquete estadístico SAS (SAS Institute, 2011), del cual se utilizó el procedimiento GLM para realizar el análisis de varianza y se aplicó la prueba de Tukey para realizar el ajuste de diferencia entre medias. Una prueba de contrastes ortogonales fue ejecutada para comparar resultados en las variables de respuesta con la inclusión de saborizantes y la ausencia de los mismos (control). La significancia de los tratamientos fue declarada cuando $\mathrm{P}<0,05$ y las tendencias a $\mathrm{P}<0,10$. 


\section{Resultados}

Los resultados obtenidos indicaron que en el consumo de pasto king grass que realizaron los caprinos, no se vio afectado por la inclusión de los agentes saborizantes; sin embargo, al comparar los sabores entre sí, fue posible observar que los animales utilizados en este experimento tuvieron una preferencia gustativa por ciertos sabores aplicados.

El mayor consumo de forraje en base seca fue de $0,977 \mathrm{~kg} /$ día y se observó cuando el pasto se trató con extracto comercial de café, que le generó un sabor amargo. Este último tratamiento no mostró diferencias significativas $(\mathrm{P}>0,10)$ con respecto al sabor salado $(0,922 \mathrm{~kg} \mathrm{MS} / \mathrm{d})$, pero el consumo si difirió cuando se comparó con el tratamiento que produjo el sabor dulce $(0,885 \mathrm{~kg} \mathrm{MS} / \mathrm{d})(\mathrm{P}<0,05)$. Cuando el forraje fue tratado con los saborizantes dulce y salado, no se apreciaron diferencias significativas $(\mathrm{P}>0,10)$ en la ingesta diaria de materia seca.

Las cabras presentaron una menor ingesta de forraje en base seca cuando el material fue mezclado con ácido cítrico (sabor ácido), donde el consumo promedio fue de 0,797 kg de MS/cabra/d (Cuadro 2). Las diferencias entre el sabor ácido y los demás sabores fueron significativas $(\mathrm{P}<0,05)$.

La cantidad de materia seca que requiere un animal se estima en función de su peso vivo (NRC, 1981), en este trabajo en promedio de todos los tratamientos, los animales presentaron una ingesta de materia seca equivalente al 1,98\% de su peso vivo, mientras que, el consumo en base fresca fue equivalente al $11 \%$ de su peso corporal; no obstante, ese consumo fue exclusivamente del forraje. Dado que este parámetro está estrechamente ligado al consumo, las diferencias entre tratamientos fueron similares a las observadas para el consumo en base seca, los sabores ácido y amargo fueron los que más difirieron entre ellos, y esa diferencia fue de alrededor de un $22 \%$. Entre dulce y salado no se observaron diferencias significativas, pero entre dulce y amargo la diferencia fue de un 9\%. Acá tampoco fue posible observar un efecto de la concentración ni efectos de la interacción entre las concentraciones y las fuentes de sabor.

Al evaluar diferentes concentraciones de los diferentes modificadores de sabor, no fue posible encontrar diferencias estadísticas $(\mathrm{P}>0,1)$ para asegurar que el consumo de materia seca se vio favorecida por un aumento en la intensidad de los sabores (Cuadro 3). Además, tampoco se observaron efectos cuando se valoró la interacción entre la fuente de sabor y la concentración del mismo.

Con respecto al porcentaje de aceptación, los animales tuvieron una mayor aceptación por el sabor amargo, al consumir alrededor de un $68 \%$ del total del material que se les ofreció, mientras que, con los tratamientos dulce y salado el porcentaje de aceptación fue de $62 \%$. Por su parte, cuando el king grass se combinó con el saborizante ácido las cabras mostraron menor aceptación, la diferencia con respecto al sabor de mayor preferencia (amargo) fue de un $12 \%$.

Los animales fueron alimentados a tres diferentes tiempos: 08:00 am, 10:30 am y 01:30 pm; y los consumos de materia seca observados fueron $0,280 \mathrm{~kg}, 0,319$ y $0,296 \mathrm{~kg}$, respectivamente. La ingesta que mostraron los animales a las 10:30 am fue significativamente diferente con respecto a los otros dos tiempos, más estos no difirieron entre sí.

\section{Discusión}

Estimular el consumo de materia seca en los caprinos tiene como objetivo aportar la mayor cantidad de nutrientes para no restringir la capacidad productiva del animal (Görgülü et al., 2008), no obstante, en el caso del componente forrajero existen factores propios de las plantas (madurez, composición química y morfología) que pueden influenciar la ingesta, debido al efecto que tienen sobre la palatabilidad del forraje (Kaitho et al., 1996; Morand-Fehr, 2003), y la inclusión de modificadores del sabor es una alternativa para enmascarar el efecto de esos aspectos intrínsecos. 
Cuadro 2. Consumo de materia verde (MV), consumo de materia seca (MS), grado de aceptación y consumo de materia verde y seca como porcentaje del peso vivo observado, cuando los caprinos fueron alimentados con pasto Pennisetum purpureum cv king grass cosechado a 75 días y tratado con diferentes sabores. Ochomogo, Cartago, Costa Rica. 2015.

Table 2. Fresh matter intake (MV), dry matter intake (MS), degree of acceptance and intake of fresh matter and dry mater as a percentage of body weight observed when goats were fed with Pennisetum purpureum cv King grass harvested at 75 days and treated with different flavors. Ochomogo, Cartago, Costa Rica. 2015.

\begin{tabular}{lcccccccccc}
\hline Sabor & $\begin{array}{c}\text { Consumo MV1, } \\
\mathbf{k g} / \mathbf{d}\end{array}$ & \multicolumn{2}{c}{$\begin{array}{c}\text { Consumo } \\
\text { MS2, kg/d }\end{array}$} & \multicolumn{2}{c}{$\begin{array}{c}\text { Aceptación, } \\
\text { \% }\end{array}$} & \multicolumn{2}{c}{$\begin{array}{c}\text { Consumo MV, } \\
\text { \% PV3 }\end{array}$} & $\begin{array}{c}\text { Consumo MS, } \\
\text { \%PV }\end{array}$ \\
\hline Ácido & 4,56 & A & 0,797 & A & $56,43 \%$ & A & 10,17 & A & 1,77 & A \\
Amargo & 5,58 & B & 0,977 & B & $68,26 \%$ & B & 12,44 & B & 2,16 & B \\
Dulce & 5,10 & C & 0,885 & C & $61,83 \%$ & C & 11,31 & C & 1,97 & C \\
Salado & 5,29 & BC & 0,922 & BC & $62,96 \%$ & C & 11,76 & BC & 2,05 & BC \\
\hline Promedio & 5,13 & & 0,895 & $62,37 \%$ & 11,42 & & 1,98 & \\
EE4 & 0,129 & & 0,024 & 4,50 & 0,30 & & 0,051 \\
\hline
\end{tabular}

${ }^{1}$ Materia verde / Fresh matter.

${ }^{2}$ Materia seca / Dry matter.

${ }^{3}$ Peso vivo / Body weigth.

${ }^{4}$ Error estándar / Standard error.

Letras diferentes indican diferencias significativas $\mathrm{P}<0,05$ / Different letters indicates significant differences $\mathrm{P}<0,05$.

Cuadro 3. Consumo de materia verde (MV), materia seca (MS), grado de aceptación y consumo de materia verde y seca, como porcentaje del peso vivo observado, cuando animales caprinos fueron alimentados con pasto Pennisetum purpureum cv king grass cosechado a 75 días y tratado con diferentes concentraciones de sabores. Ochomogo, Cartago, Costa Rica. 2015.

Table 3. Fresh matter intake (MV), dry matter intake (MS), degree of acceptance and intake of fresh matter and dry mater as a percentage of body weight observed in goats when they were fed with Pennisetum purpureum cv King grass harvested at 75 days and treated with different flavor concentrations. Ochomogo, Cartago. 2015.

\begin{tabular}{cccccc}
\hline Concentración & Consumo MV & Consumo MS & Aceptación, \% & $\begin{array}{c}\text { Consumo MV, } \\
\text { \%PV }\end{array}$ & $\begin{array}{c}\text { Consumo MS, } \\
\text { \% PV }\end{array}$ \\
\hline 0,00 & 5,22 & 0,908 & 63,33 & 11,59 & 2,02 \\
0,25 & 5,24 & 0,911 & 63,51 & 11,63 & 2,02 \\
0,35 & 4,92 & 0,856 & 59,77 & 10,94 & 1,90 \\
0,75 & 5,15 & 0,897 & 62,65 & 11,46 & 1,99 \\
1,00 & 5,17 & 0,900 & 62,84 & 11,49 & 2,00 \\
\hline Promedio & 5,14 & 0,894 & 62,42 & 11,42 & 1,98 \\
EE* & 0,170 & 0,030 & 1,90 & 0,38 & 0,650 \\
\hline
\end{tabular}

*EE = error estándar / Standard error.

Los resultados obtenidos en relación con la ausencia de diferencias significativas entre el consumo de materia seca del material tratado con sabores artificiales $v s$ el tratamiento control, se asemejan a los resultados obtenidos por Nolte y Provenza (1992), quienes evaluaron la adición de sabores naturales de extractos de cebolla y ajo a una mezcla de alfalfa y cebada, estos autores mencionan que los animales sometidos a los modificadores de sabor 
consumieron cantidades de materia seca similares a los que fueron sometidos al tratamiento sin sabor. Tampoco se observaron diferencias cuando desarrollaron cuatro experimentos donde utilizaron pellets de alfalfa mezclados con compuestos volátiles (eugenol, $\alpha$-terpineol, terpin-4-ol o metil eugenol) para medir el consumo en ovejas (Estell et al., 2007). Sin embargo, Robertson et al. (2006) sí observaron diferencias significativas entre el grupo control y los animales que consumieron alimento saborizado con agentes sintéticos agregados al agua (caramelo y maple para lograr sabor dulce; ajo, trufa y cebolla como alternativas saladas; y por último naranja, fresa y manzana), solamente que en este, los tratamientos fueron aplicados a pellets de cebada. Estos resultados son un indicativo de que los caprinos alimentados con el king grass utilizado en este trabajo, basaron su consumo en llenar los requerimientos nutricionales de materia seca más que en un estímulo sensorial. De acuerdo con algunas fuentes, cuando el material es de alta calidad con un contenido de proteína cruda que pasa el 8\%, y con un porcentaje de fibra en detergente neutro menor a un 40\%, estimula el consumo de materia seca que efectúan las cabras (Quiroz-Cardoso et al., 2015); sin embargo, en el caso del king grass el contenido proteico es menor que eso y además su contenido fibroso supera el parámetro descrito anteriormente, lo que podría limitar la ingesta por la baja digestibilidad del material (Hernández et al., 2013). Es importante mencionar que el efecto de los saborizantes depende de la palatabilidad basal de los materiales a alimentar (Baumont, 1996), la ausencia de diferencias significativas entre los tratamientos y el control, sugieren que las características nutricionales del pasto cosechado a esa edad ocasionaron que el forraje fuera lo suficientemente palatable como para no manifestar respuesta a la aplicación de un saborizante.

Al comparar los cuatro sabores (amargo, salado, dulce y ácido) entre sí, se observó que el consumo de materia seca y en el porcentaje de aceptación del forraje mostrado por los caprinos, fue menor cuando se incluyó la fuente de sabor ácido, lo cual podría limitar las respuestas productivas a materiales con procesos fermentativos como los ensilajes, ya que algunos trabajos mencionan que a pesar de la digestibilidad y del alto valor nutritivo de ensilajes, el aprovechamiento en cabras es limitado con un bajo consumo voluntario (Tjandraatmadja et al., 1993; Hussain et al., 1996).

Algunos reportes indican que los rumiantes, en general, tienen preferencias por los alimentos dulces, debido a que se asocia el sabor con un mayor contenido energético (Lamy et al., 2012), no obstante, algunos endulzantes estimulan la secreción de insulina y aumentan el apetito, a pesar de que su contenido calórico sea bajo. Las cabras usadas en este estudio, a pesar de que el sabor dulce no fue el de mayor preferencia, si tuvieron mayor aceptación que el sabor ácido, lo que sugiere que los caprinos a pesar de que el saborizante pudo ser reconocido por las papilas gustativas fungiformes y caliciformes, y estimular los quimiorreceptores presentes en la lengua (Bell y Kitchell, 1966; Fonseca et al., 2011), la concentración energética del saborizante fue muy baja como para generar una respuesta metabólica que estimulara el consumo.

Las proteínas receptoras encargadas de percibir el sabor amargo (T2R), se encuentran localizadas en la superficie de las papilas gustativas, específicamente en las células receptoras del sabor (Ferreira et al., 2015). A pesar de que este sabor es comúnmente asociado con la presencia de compuestos tóxicos y taninos que pueden desencadenar efectos contraproducentes en los caprinos (De-Rosa et al., 2002; Bhandari y Kawabata, 2005), la mayor ingesta de materia seca y el mayor porcentaje de aceptación que se observó con el sabor amargo, podría sugerir que no necesariamente el efecto del sabor es el que le indica al animal la presencia de estos metabolitos de las plantas, ya que en la fuente forrajera utilizada no hay reportes de la presencia de dichos compuestos.

Por otro lado, con el saborizante utilizado para generar el sabor ácido, es posible que exista una interacción con la saliva, la cual tiene la habilidad de cambiar la percepción sensorial de los alimentos al entrar en contacto con algunos de sus compuestos (Lamy et al., 2012).

Las diferencias en cuanto a preferencias pueden verse influenciadas por la producción de algún aroma por parte de los saborizantes; sin embargo, es difícil hacer una separación de las respuestas que son producidas por el olor y cuales por el sabor (Baumont, 1996). 
El promedio de todos los tratamientos para el parámetro consumo de materia seca como porcentaje del peso vivo del animal, fue de $1,98 \%$, valor menor al 2,5\% observado en un trabajo donde las cabras fueron alimentadas también con king grass cosechado a tres diferentes edades (Chacón-Hernández y Vargas-Rodríguez, 2010), y a otro trabajo (Rodrigues et al., 2013), que indica que las cabras comieron alrededor del 2,75\% de su peso corporal, cuando fueron alimentadas con Panicum maximum cv. Tanzania. No obstante, en ambos estudios el parámetro reportado contempló tanto el suministro de concentrado como el forraje, mientras que, el consumo realizado en el presente trabajo fue proveniente solamente del forraje.

\section{Conclusiones}

El efecto de modificadores de sabor depende del material sobre el cual se aplica, en el caso del Pennisetum purpureum cv king grass no se justificó su aplicación.

Al comparar distintos saborizantes se observó que los caprinos prefieren materiales con sabor amargo y fueron menos receptivos al sabor ácido.

La concentración de sabor en este estudio no afectó el consumo de materia seca.

\section{Literatura citada}

Animut, G., A. Goetsch, G. Aiken, R. Puchala, G. Detweiler, C. Krehbiel, R. Merkel, T. Sahlu, L. Dawson, and Z. Johnson. 2005. Grazing behavior and energy expenditure by sheep and goats co-grazing grass/forb pastures at three stocking rates. Small Rumin. Res. 59:191-201. doi:10.1016/j.smallrumres.2005.05.014

AOAC (Association of Official Agricultural Chemists). 1984. Official methods of analysis. Method 7.033-7.037. 14th ed. AOAC Int., Arlington, VA, USA.

AOAC (Association of Official Agricultural Chemists). 2000. Official methods of analysis. Method 920.9. 17th ed. AOAC Int., Arlington, VA, USA.

Araujo, J., and N. Milgram. 2004. A novel cognitive palatability assessment protocol for dogs. J. Anim. Sci. 82:2200-2206. doi: $10.2527 / 2004.8272200 x$

Araya-Mora, M., y C. Boschini-Figueroa. 2005. Producción de forraje y calidad nutricional de variedades de Pennisetum purpureum en la meseta central de Costa Rica. Agron. Mesoam. 16:37-43. doi:10.15517/am.v16i1.5180

Baumont, R. 1996. Palatability and feeding behaviour in ruminants. Areview. Ann. Zootech. 45:385-400. doi:10.1051/ animres:19960501

Bell, F.R., and R.L. Kitchell. 1966. Taste reception in the goat, sheep and calf. J. Physiol. 183:145-151.

Bhandari, M.R., and J. Kawabata. 2005. Bitterness and toxicity in wild yam (Dioscorea spp.) tubers of Nepal. Plant Foods Hum. Nutr. 60:129-135. doi:10.1007/s11130-005-6841-1

Chacón-Hernández, P.A., y C.F. Vargas-Rodríguez. 2010. Consumo de Pennisetum purpureum cv. King grass a tres edades de cosecha en caprinos. Agron. Mesoam. 21:267-274. doi:10.15517/am.v21i2.4888

De-Rosa, G., L. Moio, F. Napolitano, F. Grasso, L. Gubitosi, and A. Bordi. 2002. Influence of flavor on goat feeding preferences. J. Chem. Ecol. 28:269-281. doi:10.1023/A:1017977906903

Estell,R.,E. Fredrickson, D.Anderson, and M. Remmenga. 2007. Effects of eugenol, $\alpha$-terpineol, terpin-4-ol, and methyl eugenol on consumption of alfalfa pellets by sheep. Small Rumin. Res. 73:272-276. doi:10.1016/j.smallrumres.2006.12.008 
Ferreira, A.M., A.T. Marques, M. Bhide, V. Cubric-Curik, K. Hollung, C.H. Knight, K. Raundrup, J. Lippolis, M. Palmer, and E. Sales-Baptista. 2015. Sequence analysis of bitter taste receptor gene repertoires in different ruminant species. PLoS One 10:e0124933. doi:10.1371/journal.pone.0124933

Fonseca, E.T.d., C.M.d. Oliveira, A.L.R. Franciolli, e M.A. Miglino. 2011. Características das papilas o dorso da língua de cabras (Capra hircus): Estudo por de microscopia eletrônica de varredura e luz. Pesqui. Vet. Bras. 31:67-73. doi:10.1590/ S0100-736X2011001300011.

Gallego-Calvo, L., M. Gatica, J. Guzmán, and L. Zarazaga. 2014. Role of body condition score and body weight in the control of seasonal reproduction in blanca andaluza goats. Anim. Reprod. Sci. 151:157-163. doi:10.1016/j.anireprosci.2014.10.011

Görgülü, M., M. Boğa, A. Şahin, U. Serbester, H.R. Kutlu, and S. Şahinler. 2008. Diet selection and eating behavior of lactating goats subjected to time restricted feeding in choice and single feeding system. Small Rumin. Res. 78:41-47. doi:10.1016/j. smallrumres.2008.04.004

Hernández, S.R., J.O. Pérez, I.G. Segura, R.J. Guillén, F.L. López, and A.C. Izquierdo. 2013. Use of Crescentia alata and Guazuma ulmifolia fruits in lamb feeding in subtropical region of Guerrero, Mexico. Rev. Cient. FCV-LUZ 23:157-162.

Hussain, Q., Ø. Havrevoll, and L. Eik. 1996. Effect of type of roughage on feed intake, milk yield and body condition of pregnant goats. Small Rumin. Res. 22:131-139. doi:10.1016/S0921-4488(96)00868-1

Kaitho, R., N. Umunna, I. Nsahlai, S. Tamminga, J. Van-Bruchem, J. Hanson, and M. Van-De-Wouw. 1996. Palatability of multipurpose tree species: Effect of species and length of study on intake and relative palatability by sheep. Agrofor. Syst. 33:249-261. doi:10.1007/BF00055426

Lamy, E., F. Capela-e-Silva, A. Ferreira, and E.S. Baptista. 2012. The influence of oral environment on diet choices in goats: A focus on saliva protein composition. In: D.E. Garrote, and G.J. Andrade, Goats: Habitat, breeding and management. Nova Science Publishers Inc., NY, USA. p. 93-111.

Márquez, F., J. Sánchez, D. Urbano, y C. Dávila. 2007. Evaluación de la frecuencia de corte y tipos de fertilización sobre tres genotipos de pasto elefante (Pennisetum purpureum). 1. Rendimiento y contenido de proteína. Zootec. Trop. 25:253-259.

Miller-Cushon, E., M. Terré, T. DeVries, and A. Bach. 2014. The effect of palatability of protein source on dietary selection in dairy calves. J. Dairy Sci. 97:4444-4454. doi:10.3168/jds.2013-7816

Morand-Fehr, P. 2003. Dietary choices of goats at the trough. Small Rumin. Res. 49:231-239. doi:10.1016/S09214488(03)00141-X

NRC (National Research Council). 1981. Nutrient requirements of goats: Angora, dairy, and meat goats in temperate and tropical countries. National Academies Press, WA, USA.

Nolte, D.L., and F.D. Provenza. 1992. Food preferences in lambs after exposure to flavors in solid foods. Appl. Anim. Behav. Sci. 32:337-347. doi:10.1016/S0168-1591(05)80030-9

Olivares, J., S. Rojas, I. Gutiérrez, E.J. Míreles, M.T. Valencia, F. Quiroz, y N. Forrajes. 2012. Uso del fruto de tres leguminosas arbóreas en pruebas de cafetería en rumiantes en el trópico de Guerrero México. En: A. Romo-Rubio et al., editores, Reunión nacional de cuerpos académicos del área de la medicina veterinaria y zootecnia 62. UASCA, Sinaloa, MEX. p. 55-60.

Pineda-Cordero, L., P. Chacón-Hernández, y C. Boschini-Figueroa. 2016. Evaluación de la calidad del ensilado de pasto estrella africana (Cynodon nlemfluensis) mezclado con tres diferentes aditivos. Agron. Costarricense 40:11-27.

Quiroz-Cardoso, F., S. Rojas-Hernández, J. Olivares-Pérez, E. Hernández-Castro, R. Jiménez-Guillén, A. Córdova-Izquierdo, A. Villa-Mancera, and S. Abdel-Fattah. 2015. Composición nutricional, consumo e índices de palatabilidad relativa de los frutos de tres acacias en la alimentación de ovejas y cabras. Arch. Med. Vet. 47:33-38. doi:10.4067/S0301732 X2015000100007 
Rangel, G., C. González, L. Novoa, E. Hurtado, y H. Vecchionacce. 2004. Estudios de aceptabilidad de harina de follajes tropicales como recursos alternativos en dietas para cerdos. Albéitar. http://albeitar.portalveterinaria.com/noticia/3457/ articulos-porcino-archivo/estudios-de-aceptabilidad-de-harina-de-follajes-tropicales-como-recursos-alternativos-endietas-para-cerdos.html (consultado el 14 de agosto del 2018).

Robertson, E., I.J. Gordon, and F.J. Pérez-Barbería. 2006. Preferences of sheep and goats for straw pellets treated with different food-flavouring agents. Small Rumin. Res. 63:50-57. doi:10.1016/j.smallrumres.2005.02.007

Rodrigues, M.M., M.E. de-Oliveira, R.L. de-Moura, R.M.O. Alves, W.K.A. Silva, and M.P. Cortez. 2013. Forage intake and behavior of goats on Tanzania-grass pasture at two regrowth ages. Acta Sci., Anim. Sci. 35:37-41. doi:10.4025/ actascianimsci.v35i1.16035

Rutter, S.M. 2006. Diet preference for grass and legumes in free-ranging domestic sheep and cattle: Current theory and future application. Appl. Anim. Behav. Sci. 97:17-35. doi:10.1016/j.applanim.2005.11.016

SAS Institute. 2011. SAS/STAT 9.3 user's guide. SAS Institute Inc., Cary, NC, USA.

Taxonomy-Soil. 2003. Keys to soil taxonomy. US Department of Agriculture, NRCS, SW, and USDA, WA, USA.

Tjandraatmadja, M., I.C. MacRae, and B.W. Norton. 1993. Intake and digestibility of sorghum silage by goats. Anim. Feed Sci. Technol. 41:171-179. doi:10.1016/0377-8401(93)90010-H

Van-Soest, P.J., J.B. Robertson, and B.A. Lewis. 1991. Methods for dietary fiber, neutral detergent fiber, and nonstarch polysaccharides in relation to animal nutrition. J. Dairy Sci. 74:3583-3597. doi:10.3168/jds.S0022-0302(91)78551-2

Vargas-Rodríguez, C.F. 2007. FAMACHA@, control de haemonchosis en caprinos. Agron. Mesoam. 17:79-88. doi:10.15517/ am.v17i1.5069 